\title{
SCREENING OF ANTENATAL MOTHERS AND PREVENTION OF PERINATAL GROUP B STREPTOCOCCAL INFECTION
}

\author{
Raj M, Razali N, Sulaiman S
}

Department of Obstetrics and Gynaecology, Faculty of Medicine, University of Malaya, Kuala Lumpur

\begin{abstract}
:
The objectives of this study were: (1) to detect group B streptococcus (GBS) carriers among antenatal patients during pregnancy ( 35 to 37 weeks) in the University Malaya Medical Centre (UMMC), Kuala Lumpur, Malaysia, and (2) to give antibiotic prophylaxis during the intrapartum period for the GBS carrier patients. The aim is to prevent early onset of GBS infection in newborn. This pilot study was carried out from 1st July 2005 to 31st August 2005. It is a prospective study involving 56 pregnant women who were seen at the antenatal clinic in the UMMC between 35 to 37 weeks gestation. High vaginal swab and low vaginal together with endoanal swab were taken for culture and sensitivity. The antibiotic prophylaxis was started early in the intrapartum period for patients with positive culture for GBS. GBS was detected in 18 patients which contributes to about $32 \%$ of the study population. The proper choice of antibiotic is important in successful disease prevention. (JUMMEC 2008; 12 (1): 27-30)
\end{abstract}

KEYWORDS: group B streptococcus, screening, pregnant women

\section{Introduction}

Streptococcus agalactiae, the causative organism of Group B streptococcus (GBS) disease is a gram-positive diplococcus believed to be a bovine pathogenic organism responsible for mastitis in cattle and it was not until 1938 when Fry described three patients with fatal puerperal sepsis that GBS was known to cause human infection (1).

Over the next two decades, only sporadic cases of GBS infection were reported and most clinicians were unaware of GBS and its sequelae. In the 1960s GBS was found to be responsible for more perinatal morbidity and mortality than had been previously appreciated (2). During the 1970s researchers from geographically diverse regions began reporting a dramatic increase in the incidence of neonatal septicemia and meningitis caused by GBS; to the extent that GBS replaced Escherichia coli as the leading cause of bacteremia and meningitis in the neonatal period ( 3 ).

The rates of GBS disease have dramatically declined primarily because of the discovery in the early 1980s that administering antibiotics to at risk women during labour could prevent invasive disease in the first week of life (4).
Numerous studies have documented that the timing of prenatal screening cultures, the anatomic sites swab and the precise biologic methods used for culture and detection of GBS can affect accuracy of identifying colonization status. It is recommended that all pregnant women undergo bacteriological screening with vaginal and rectal swabs taken for GBS culture at 35 to 37 weeks of gestation $(5,6)$.

\section{Materials and method}

This study was carried out from 1st July, 2005, to 31st August, 2005. It was a prospective pilot study involving pregnant women between 35 and 37 weeks' gestation, who were seen at the antenatal clinic in the University Malaya Medical Centre (UMMC), Kuala Lumpur, Malaysia. An explanation was given to the patient regarding the benefits of prenatal screening for GBS

Correspondence:

Sofiah Sulaiman

Department of Obstetrics and Gynaecology

Faculty of Medicine, University of Malaya

50603 Kuala Lumpur

Email: drsofie@hotmail.com 
colonization in pregnancy. Consent was obtained once the patient agreed to participate in the study. Some fifty-six (56) pregnant woman participated in the study. Women with GBS bacteriuria in any concentration during their current pregnancy or who previously gave birth to an infant with GBS disease were not included in the GBS screening programme.

Each patient had two swabs taken at the same time, a high vaginal swab and a low vaginal together with endoanal swab. Ethic approval was obtained from the UMMC ethic committee.

\section{Method for culturing Group B Streptococci}

The first swab was taken from the lower part of the vagina and anus before it was placed into the culture medium. Subsequently, a Cusco speculum was introduced and a high vaginal swab was taken and placed into another culture medium. The culture medium used in this study was non nutritive transport medium (Amies media without charcoal) and the samples were sent to the laboratory on the same day. Women with positve cultures for GBS were given intrapartum antimicrobial prophylaxis. Penicillin $\mathrm{G}$ or Ampicillin is the antibiotic of choice. Should the patient be allergic to penicillin, the Centers for Disease Control and Prevention (CDC) suggests using clindamycin or erythromycin parenterally. The data were collected in a data sheet after delivery and analysed.

\section{Results}

The study participants reflected the composition of the low risk antenatal group at UMMC in their age, gravidity and parity (Table 1 ). More than half of the study population (59\%) are multigravidas.

Among the multigravid women, $6 \%$ of them had GBS infection documented in the previous pregnancy. The status of GBS infection in previous pregnancy was not known in $66.7 \%$ of the multigravid women. This was because there were no previous screening performed or the women had not delivered in UMMC previously. Out of the 56 participants, three went into preterm labour and two of them were GBS carriers. There were 18 patients who were found to have positive results for GBS and six of them had some vaginal discharge during that pregnancy and eleven of them were primigravidas.
Table 1: Characteristics of participants

\begin{tabular}{ll}
\hline Characteristics & Frequency (\%) \\
\hline Age Mean ( \pm sd) & $29.9( \pm 4.0)$ \\
$\quad$ Age group in years & $49 / 56(87.5)$ \\
$21-34$ & $7 / 56(12.5)$ \\
$>35$ & \\
Parity & $23 / 56(41.0)$ \\
0 & $30 / 56(53.6)$ \\
$1-3$ & $3 / 56(5.4)$ \\
$>4$ & \\
Presence of vaginal discharge in & \\
current pregnancy (n=56) & $19 / 56(33.9)$ \\
Women with vaginal discharge & $37 / 56(66.1)$ \\
Women without vaginal discharge & $18 / 56$ \\
Positive result & $6 / 18(33.3)$ \\
Present of vaginal discharge & $11 / 18(61.1 \%)$ \\
Primigravida & \\
Occupation & $13 / 56(23.2)$ \\
Housewife & $19 / 56(33.9)$ \\
Clerical & $24 / 56(42.9)$ \\
Professional &
\end{tabular}

About two thirds (66.1\%) of the study population were asymptomatic and it was found that $32 \%$ of them had positive culture for GBS. Results of positive GBS culture from different swabs culture site are shown in Table 2.

Table 2: Results of the culture for HVS and LVS with endoanal swab

\begin{tabular}{lcc}
\hline Results/site & $\mathbf{n}$ & $\mathbf{( \% )}$ \\
\hline Detected both & 11 & 19.6 \\
Not detected & 29 & 51.8 \\
HVS alone & 2 & 3.6 \\
LVS with anorectal alone & 5 & 8.9 \\
Others & 9 & 16.1 \\
\hline Total & 56 & 100 \\
\hline
\end{tabular}

Most positive cultures were obtained from combination of HVS and LVS together with anorectal swab (19.6\%). Combined LVS and anorectal swab resulted in $8.9 \%$ positive cultures compare to HVS alone (3.6\%).

All the positive GBS cultures were reported to be sensitive to Penicillin and Ampicillin ( $n=18,100 \%)$. The sensitivity to other antibiotics were as such, Cephalexin ( $n=14,78 \%)$, Erythromycin ( $n=13,72 \%$ ) and Cefuroxime ( $n=8,44 \%)$. Ampicillin was used in 
all the positive carrier mothers and the dose was initially $2 \mathrm{gm}$ intravenously followed by $1 \mathrm{gm}$ every four hours.

Out of 56 patients, 43 of them had spontaneous vaginal deliveries and 13 had caesarean sections. Among the GBS carrier patients, two of them had emergency sections done due to poor progress and they had intrapartum antibiotic prophylaxis.

None of the neonates were detected with early onset group B streptococcus sepsis (EOGBS). Out of 56 deliveries, two babies were admitted to Special Care Nursery $(\mathrm{SCN})$, where one of them had cardiac abnormality. The other baby was admitted due to low apgar secondary to abruptio placenta (emergency cesarean section was performed at 38 weeks).

\section{Discussion and conclusion}

GBS is one of the leading cause of neonatal morbidity and mortality. The fetus could be infected during pregnancy leading to fetal death or acquired the infection during passage through the birth canal when the mother's vagina is colonised with this bacteria. Colonisation of GBS in pregnant women were found in many studies to be between $10 \%$ and $30 \%(6,7)$ and it was found to be higher in pregnant women who had complicated pregnancy (7). In this study, it was found to be positive in $32 \%$ of the population studied.

In 2002, CDC, the American College of Obstetricians and Gynecologists (ACOG) and the American Academy of Pediatrics (AAP) issued revised guidelines for prevention of early-onset GBS disease (i.e., in infants aged < seven days). These guidelines recommended universal screening of all pregnant women for rectovaginal GBS colonization at 35-37 weeks' gestation and administration of intrapartum antibiotic prophylaxis to carriers (8). Screening for GBS was not offered in our hospital and during the duration of study period; patients who consented to the study were screened and intrapartum ampicillin were administered if they were found to be carriers of GBS. Patients who had negative culture for GBS were not given intrapartum ampicillin as prophylaxis and this practice is consistent with the recommendation by $C D C$ (9). Among the patients who were in this study, none of the babies had been infected or were detected with EOGBS. This study involved a small proportion of antenatal patients in our hospital. However, it has been reported recently that despite marked success with universal screening, GBS continues to be an important cause of early-onset sepsis and thus remains a significant public health issue (10).

The vaginal colonisation of GBS varies during the period of gestation and screening earlier than six weeks before delivery may not be a true reflection at delivery and may not accurately predict the vaginal colonisation at delivery. Cultures that were obtained between one and five weeks before delivery has sensitivity of $87 \%$ and specificity of $97 \%$ (11). In this study the cultures were taken five weeks before delivery as per CDC guideline. When cultures are obtained more than five weeks before delivery, there is a greater chance that the results will not accurately predict colonization status at delivery (12).

Other factors that have been shown to increase the recovery rate of GBS include the culture site and method of culture. A combination of vaginal and rectal swab has been reported to increase the recovery rate of GBS by $25 \%$ (13). Similarly, our study showed that recovery rate of GBS was highest when the culture were obtained from combination of vaginal and rectal swab (19.6\%) compared to HVS alone (3.6\%). In our study, the Amies medium, which is a nonselective transport medium was used which led to a recovery rate of $30 \%$. It has been recommended to use a selective broth medium that enhances the growth of GBS and also supplemented with antibiotics to inhibit the growth of other organisms (8). The use of this selective medium has been shown to increase the GBS recovery rate by $50 \% .(13)$

The appropriate choice of antibiotics is an important factor to prevent perinatal infection. The CDC guidelines recommended the use of Penicillin as the first choice and Ampicillin as the second choice. Parenteral Erythromycin or Clindamycin are the suggested alternatives if the patient is allergic to Penicillin (13). GBS isolates remain universally susceptible to penicillin and ampicillin as seen in our study $(100 \%)$. In our centre, most of the GBS positive carriers received ampicillin.

This study can contribute to the development of preventice strategies in that it provides evidence for 
optimal timing to obtain swabs and the antibiotic of choice to treat women who were carriers of GBS. The limitation of the study is that only a small number of women were willing to participate in the study. A bigger number of patients are needed to participate in the study to achieve the screening target. Therefore, adequate information regarding GBS disease should be disseminated to patients as well as the medical staffs.

\section{References}

1. Fry, RM. Fatal infections by hemolytic streptococcus group B. Lancet 1938; 1: 199-201.

2. Eickhoff, T., Klein JO, Daly AK, Ingall D, Finland M. Neonatal sepsis and other infections due to group B beta-hemolytic streptococci. N Engl. J Med 1964; 271: 1221-1228.

3. McCracken GH. Group B streptococci: The new challenge in neonatal infections. J Pediatr 1973; 82: 703-706.

4. Benitez WE, Gould JB, Druzin ML. Antimicrobial prevention of early-onset group $B$ streptococcal sepsis: Estimates of risk reduction based on a critical literature review. Pediatrics 1999; 103: 78.

5. Brozanski BS, Jones, JG, Krohn, MA, Sweet RL. Effect of a screening-based prevention policy on prevalence of early-onset group B streptococcal sepsis. Obstet Gynecol 2000; 95: 496-501.

6. Schuchat A, Wenger JD. Epidemiology of group B streptococcal disease: Risk factors, prevention strategies and vaccine development. Epidemiol Rev 1994; 16: 374-402.
7. Strus M, Pawlik D, Brzychczy-Wloch $M$ et al. Group B streptococcus colonization of pregnant women and their obstetric and neonatal ward of the University Hospital in Krakow, Poland. J Med Microbiol 2009 Feb; 58 (Pt2): 228-233.

8. Centers for Disease Control and Prevention. Trends in perinatal group B streptococcal disease-United States, 2000-2006. MMWR Morb Mortal Wkly Rep 2009 Feb 13; 58 (5): 109-112.

9. de Cueto M, Sanchez MJ, Sampedro A, Miranda JA, Herruzo AJ, Rosa-Fraile M. Timing of intrapartum ampicillin and prevention of vertical transmission of group B streptococcus. Obstet Gynecol 1998; 91:112-114.

10. Koenig JM, Keenan WJ. Group B streptococcus and early-onset sepsis in hte era of maternal prophylaxis. Pediatr Clin North Am 2009 June; 56 (3): 689-708.

11. Yancey MK, Schuchat A, Brown LK, Ventura VL, Markenson GR. The accuracy of late antenatal screening cultures in predicting genital group B streptococcal colonization at delivery. Obstet Gynecol 1996; 88: 811-815.

12. Hager WD. Prevention of perinatal group B streptococcal infection: Current controversies. Obstetrics and Gynaecology 2000; 96 (1): 141-145.

13. Philipson EH, Palermino DA, Robinson A. Enhanced antenatal detection of group B streptococcal colonization. Obstet Gynecol 1995; 85: 437-439. 